\title{
Theoretical Modeling of Transport Barriers in Helical Plasmas
}

\author{
Shinichiro TODA, Kimitaka ITOH and Nobuyoshi OHYABU \\ National Institute for Fusion Science, Toki 509-5292, Japan \\ (Received 11 August 2009 / Accepted 3 February 2010)
}

\begin{abstract}
A unified transport model is proposed to study the physical mechanism for the formation of electron internal transport barriers (e-ITB) in helical plasmas and internal diffusion barriers (IDB) observed in the Large Helical Device (LHD). An e-ITB can be predicted with the effect of zonal flows (ZFs) in the low collisional regime when the radial variation in particle turbulent diffusivity is included. The transport analysis in this article shows that particle fueling induces IDB formation when the unified transport model is used in the high collisional regime. After particle fueling, a steep density gradient forms. To examine the density limit for the IDB in helical toroidal plasmas, the effect of radiation loss is included in a set of transport equations.
\end{abstract}

(C) 2010 The Japan Society of Plasma Science and Nuclear Fusion Research

Keywords: helical plasma, turbulent transport, transport barrier, radial electric field, density limit

DOI: $10.1585 /$ pfr.5.011

\section{Introduction}

It is crucial to fusion research to understand turbulence-driven transport and improved confinement (transport barriers). Many kinds of improved confinement modes in the core plasma of toroidal helical plasmas have been reported, e.g., an electron internal transport barrier (e-ITB) with a strong positive radial electric field $E_{r}$. A radial transition of $E_{r}$ was predicted to induce the e-ITB due to the shear in the radial electric field in helical plasmas. The transition of $E_{r}$ was found in the Compact Helical System (CHS), and improved confinement was observed inside the transition point for $E_{r}$ [1]. Observations of the e-ITB in Wendelstein 7-AS [2], the Large Helical Device (LHD) [3, 4], and other experiments followed [5]. In a previous study of the e-ITB [6], we have shown a reduction in heat diffusivity because of the effect of zonal flows (ZFs), which qualitatively predicts the e-ITB experimentally observed in the entire region of the strong positive $E_{r}$. However, in that study we excluded the radial and temporal changes in the particle diffusivity due to the effect of ZFs. On the other hand, an IDB in the LHD [7] was recently discovered with a strong density gradient in a super dense core (SDC) plasma when a series of pellets was injected. In this article, we present a unified transport model to explain the e-ITB and the IDB. First, we confirm the reduction in turbulent diffusivities due to $\mathrm{ZFs}$ when we include radial and temporal variation in the particle turbulent diffusivity in helical plasmas. Next, we show a theoretical model for the IDB observed in the LHD. The mechanism, which is based on transport reduction due to the shear in the radial electric field, is newly examined in terms of IDB formation. We study the temporal response of the density, temperature and radial electric field to particle fueling by pellets as an initial value problem. The steepness of the

author'se-mail: toda@nifs.ac.jp density profile is found to show self-generated evolution. After pellet ablation, the shear of the radial electric field increases, and an associated reduction in the particle diffusivity is obtained. The jump in the particle diffusivity at the radial transition point of the improved confinement region steepens after pellet fueling.

Density limit phenomena control how plasma performance can be achieved. In tokamaks, confinement and density limit properties are dictated and the density limit is approximated by the empirically derived Greenwald limit scaling [8]. The importance of plasma dynamics and the critical density near strong sources of impurities has been discussed [9]. In helical plasmas, attention has been paid to density limit phenomena, e.g., $[10,11]$. Recently, the dependence of the critical density on the edge plasma parameters has been examined in LHD plasmas [12]. Helical plasmas have an additional freedom in magnetic geometry, which is utilized to investigate transport mechanisms. Density limit phenomena in toroidal helical plasmas have been examined with the analytic point model [13]. Since the radial electric field determined by the ambipolar condition in a non-axisymmetric system is known to affect the confinement properties significantly, theoretical analysis of the density limit is necessary, including radiation loss in helical plasmas by considering the effect of the ambipolar radial electric field in a set of transport equations. To examine the density limit for thermal stability in helical plasmas, we add the term for the radiation loss rate of the energy to the temporal equation for the electron temperature in a set of one-dimensional transport equations. The radiative loss of line emission from impurity ions has a temperature dependence that the loss increases if the temperature gets lower. The combined mechanism of transport and radiation loss of the energy is discussed. The dependence of the electron temperature profile on electron heat- 
ing is studied by including the radiative cooling rate in a set of transport equations to examine the density limit in helical plasmas. The parameter dependence of the critical density is derived when the effect of the radial electric field is included. A sharp decrease in the electron temperature is shown near the edge because the radiative loss rate rapidly increases at the low temperature. Phenomena such as a transition near the edge in the $E_{r}$ profile are obtained. The value of the turbulent heat diffusivity is significantly reduced because of the steep gradient in the radial electric field near the edge.

\section{One-dimensional Model for Trans- port Equations}

A one-dimensional transport model is employed. Cylindrical coordinates are used and the $r$-axis is taken in the radial direction in a cylindrical plasma in this article. The region $0<\rho<1$ is considered, where $\rho=r / a$ and $a$ is the minor radius. The temporal equations for the density $(n)$, electron temperature $\left(T_{\mathrm{e}}\right)$ and the hydrogen ion temperature $\left(T_{\mathrm{i}}\right)$ in this article are those given in [6]. The expression for the radial neoclassical flux associated with helical-ripple trapped particles is denoted by the symbol $\Gamma_{j}^{\text {na }}$ for species $j$. For e-ITB analysis, the form given in [14] is used in this article. This form covers the region from the $v_{\mathrm{j}}$ regime to the $1 / v_{\mathrm{j}}$ regime, where $v_{\mathrm{j}}$ is the collision frequency. When performing calculations about an IDB in high density plasmas, we use the form for the radial neoclassical flux given in [15], which is available in the Pfirsch-Schlüter regime, because the plasma state of an IDB/SDC plasma corresponds to the high collisional regime. In the temporal equation for the density, the term $S_{n}$, which represents the particle source, is used. The quantity $S_{\mathrm{p}}$ is the modeled particle source of pellet fueling in the case of IDB analysis. (In e-ITB calculations, $S_{\mathrm{p}}=0$.) In the equations for the electron and ion temperatures, we have terms that represent the absorbed power as a result of electron cyclotron resonance heating (ECRH) and the absorbed power of ions. In the temporal equations for the density and temperatures, we use the turbulent particle diffusivity $D_{\mathrm{T}}$ and the turbulent heat diffusivity $\chi_{\mathrm{T}}$, respectively. A theoretical model for the turbulent diffusivity is adopted.

In calculations of the e-ITB including the effect of $\mathrm{ZFs}$, the temporal equation for the radial electric field in a nonaxisymmetric system [16] is analyzed in system of the temporal transport equations given in [6]. On the other hand, in IDB analysis, we use the ambipolar condition $\sum_{j} Z_{j} \Gamma_{j}^{\mathrm{na}}=0$ to determine the radial profile of the electric field, where $Z_{j}$ is the charge number of species $j$.

The equations of the density, temperature, and electric field are solved under the appropriate boundary conditions. We fix the boundary condition at the center of the plasma $(\rho=0)$ such that $n^{\prime}=T_{\mathrm{e}}^{\prime}=T_{\mathrm{i}}^{\prime}=E_{r}=0$. For the diffusion equation for the radial electric field, the bound- ary condition at the edge $(\rho=1)$ is set to $\sum_{j} Z_{j} \Gamma_{j}^{\mathrm{na}}=0$. The boundary conditions for the density and temperature at the edge $(\rho=1)$ are given by specifying the gradient scale lengths. We employ those that are reasonable in the LHD: $-n / n^{\prime}=-T_{\mathrm{e}} / T_{\mathrm{e}}^{\prime}=-T_{\mathrm{i}} / T_{\mathrm{i}}^{\prime}=0.1 \mathrm{~m}$. The machine parameters, which are similar to those of the LHD, are set to be $R=3.6 \mathrm{~m}, a=0.6 \mathrm{~m}, B=3 \mathrm{~T}, \ell=2$ and $m=10$. In this study, we set the safety factor and the helical ripple coefficient to $q=1 /\left(0.4+1.2 \rho^{2}\right)$ and $\epsilon_{\mathrm{h}}=2 \sqrt{1-(2 /(m q(0)-1)} I_{2}(m r / R)$, respectively. Here, $q(0)$ is the value of the safety factor at $\rho=0$ and $I_{2}$ is the second-order modified Bessel function.

\section{Model of Turbulent Transport Co- efficients}

We adopt a model for the anomalous heat diffusivity $\chi_{\mathrm{a}}$ based on the theory of self-sustained turbulence due to the interchange mode [17] and the ballooning mode[18], both driven by the current diffusivity. A reduction in anomalous transport due to an inhomogeneous radial electric field was examined in a toroidal helical system. The anomalous transport coefficient for the temperatures is given as $\chi_{\mathrm{a}}=$ $\chi_{0} /\left(1+G \omega_{\mathrm{E} 1}^{2}\right)\left(\chi_{0}=F(s, \alpha) \alpha^{3 / 2} c^{2} v_{\mathrm{A}} /\left(\omega_{\mathrm{pe}}^{2} q R\right)\right)$, where $\omega_{\mathrm{pe}}$ is the electron plasma frequency. The factor $F(s, \alpha)$ is a function of the magnetic shear $s$ and the normalized pressure gradient $\alpha$, defined by $s=r q^{\prime} / q$ and $\alpha=-q^{2} R \beta^{\prime}$. In the case of interchange mode turbulence for a system of the magnetic hill [17], we adopt the anomalous thermal conductivity $\chi_{\mathrm{a}, \mathrm{IM}}$. Details of the coefficients $F, G$ and the factor $\omega_{\mathrm{E} 1}$, which denotes the poloidal $E \times B$ rotation frequency, were given in [17] for the interchange mode. For ballooning mode turbulence (in a system with a magnetic well), we employ the anomalous thermal conductivity $\chi_{\text {a,BM. }}$. Details of the coefficients $F(s, \alpha), G$ and the factor $\omega_{\mathrm{E} 1}$ are given in [18] for ballooning mode turbulence. The greater one of these two diffusivities is adopted as $\chi_{\mathrm{a}}=\max \left(\chi_{\mathrm{a}, \mathrm{BM}}, \chi_{\mathrm{a}, \mathrm{IM}}\right)$.

We introduce the effect of ZFs to the turbulent diffusivities, because ZFs are predicted to be excited because of the strong positive electric field in the parameter regime where the e-ITB is observed. ZFs (at nearly zero frequency) are generated by fluctuations and strongly influence turbulent transport. The damping rate of ZFs, $v_{\text {damp }}$, controls turbulent transport. The damping of ZFs is caused by a collisional process and by the self-nonlinearity of ZFs [19]. Whether ZFs are excited is judged by comparing $\chi_{\mathrm{a}}$ (which is given in the absence of ZFs) with the quantity $\chi_{\text {damp }} \cong k_{\perp}^{2} q_{r}^{-2} k_{\theta}^{-2} v_{\text {damp }}$, where $q_{r}$ is the wave number of $\mathrm{ZFs}, k_{\theta}$ is the poloidal wave number of microscopic fluctuations, and $k_{\perp}$ is the perpendicular wave number of microscopic fluctuations. To determine the value of the turbulent diffusivity $\chi_{\mathrm{T}}$ with the effect of ZFs included in the transport codes, the procedure in $[6,20,21]$ is used.

Because $v_{\text {damp }}$ depends on $E_{r}$, it is small in the elec- 
tron root branch of the e-ITB (i.e., at the strong positive $E_{r}$ ). The turbulent transport coefficient decreases if a strong positive radial electric field is established in calculations [21]. The value for the turbulent diffusivities of particles is set to $D_{\mathrm{T}}=\chi_{\mathrm{T}}$ to include radial variation in the density profile. We also set $D_{\mathrm{ET}}=\chi_{\mathrm{T}}$ to examine the typical length of the electric field shear at the transition point, where $D_{\mathrm{ET}}$ is the diffusion coefficient in the diffusion temporal equation for the radial electric field.

Since a strong positive electric field cannot be obtained in the parameter regime of IDB/SDC plasmas, no significant reduction in the diffusivities is predicted due to the effect of ZFs. The value for the turbulent particle diffusivity is set to $D_{\mathrm{T}}=\chi_{\mathrm{T}}$ to examine the radial variation in the profile of the particle diffusivity $D_{\mathrm{T}}$, where a steep radial gradient in the density profile can be obtained by calculation.

\section{Model of Particle and Heat Sources}

In analysis of e-ITB plasmas, the particle source $S_{n}$ is set to $S_{n}=S_{0} \exp \left[(r-a) / L_{n}\right]$. This profile represents the peaking at the plasma edge of the particle source due to the ionization effect. A value of $3 \times 10^{22} \mathrm{~m}^{-3} \mathrm{~s}^{-1}$ is chosen for $S_{0}$ and $L_{n}$ is set to $0.1 \mathrm{~m}$. The intensity $S_{0}$ governs the average density and is taken as a control parameter to specify the density in this article. The absorbed power of electrons is set to $1 \mathrm{MW}$, and the absorbed power of ions is taken as $0 \mathrm{MW}$. The radial profiles of the electron and ion heating terms are assumed to be proportional to $\exp \left\{-[r /(0.2 a)]^{2}\right\}$ for the sake of analytical insight.

In calculations for IDB/SDC plasmas, we introduce the term $S_{\mathrm{p}}$, representing an additional particle source, to the temporal density equation to examine the effect of pellet injection, in addition to the particle source $S_{n}$ explained above. This parameter $S_{\mathrm{p}}$ has the distribution $S_{\mathrm{p}}=S_{\mathrm{p} 0} \exp \left[-\left(r / r_{\mathrm{p}}\right)^{2}\right]$ and is set to have a value from the initial time $t=0$ to $1 \mathrm{~ms}$. In other words, we set $S_{\mathrm{p} 0}=1 \times 10^{23} \mathrm{~m}^{-3} \mathrm{~s}^{-1}$ for $0<t \leq 1 \mathrm{~ms}$ and $S_{\mathrm{p} 0}=0 \mathrm{~m}^{-3} \mathrm{~s}^{-1}$ for $t>1 \mathrm{~ms}$. We set the half width of the $S_{\mathrm{p}}$ profile to $r_{\mathrm{p}}=0.2 a$. To establish the target plasma, the particle source term $S_{n}$ is also set to $S_{n}=S_{0} \exp \left[(r-a) / L_{n}\right]$. Here, the coefficient $S_{0}$ is taken as $7 \times 10^{22} \mathrm{~m}^{-3} \mathrm{~s}^{-1}$ and $L_{n}$ is set to $0.1 \mathrm{~m}$. This particle source is chosen to obtain a self-consistent steady plasma that is used as the initial condition of the next calculation including the effect of particle fueling (pellet injection). The absorbed power of electrons is set to $1 \mathrm{MW}$ and the absorbed power of ions is taken as $0 \mathrm{MW}$. The radial profiles of the electron and ion terms of the absorbed powers are set to be proportional to $\exp \left\{-[r /(0.5 a)]^{2}\right\}$.

\section{Results of the Analysis of e-ITB Plasmas}

The bifurcation of $E_{r}$ itself induces the transition of the turbulent transport in the bulk of the plasma column as well as that of the shear in the electric field. The dependence of $\chi_{\mathrm{T}}$ on the damping rate of ZFs explains the improved confinement in the e-ITB region of toroidal helical plasmas. Reduced turbulent transport in the entire region of strong positive $E_{r}$ is demonstrated. The calculation results here include the reduction in particle diffusivity due to the strong $E_{r}$ shear at the transition point and ZFs in the region inside the transition point. One-dimensional transport analysis for an LHD-like plasma has been performed, and the radial profiles of $E_{r}, T_{\mathrm{e}}, T_{\mathrm{i}}$ and $n$ are obtained. In this analysis, the total thermal diffusivity is given as the sum of $\chi_{T}$ and the neoclassical transport diffusivity. An example is taken from a plasma sustained by ECRH. To set the lineaveraged temperature of electrons to around $\bar{T}_{\mathrm{e}}=1.1 \mathrm{keV}$ [ $T_{\mathrm{e}}$ at the center, $\left.T_{\mathrm{e}}(0)=2.6 \mathrm{keV}\right]$ and the line-averaged density to around $\bar{n}=2 \times 10^{19} \mathrm{~m}^{-3}$ for anomalous transport coefficients chosen above, the line-averaged ion temperature is chosen to be about $\bar{T}_{\mathrm{i}}=0.6 \mathrm{keV}\left[T_{\mathrm{i}}(0)=\right.$ $0.9 \mathrm{keV}]$. When we evaluate $\chi_{\text {damp }}$, we employ an estimate of $k_{\perp}^{2} q_{r}^{-2} k_{\theta}^{-2} \rho_{i}^{-2} \sim 50$ [21]. In the parameter regime examined here, we obtain strong positive $E_{r}$ in the core region: $E_{r} \simeq 15 \mathrm{kV} / \mathrm{m}$.

Figure 1 (a) shows self-consistent stationary radial profiles of the electric field without (dashed line) and with (solid line) the effect of ZFs. In both cases with and without ZFs, a radial transition of $E_{r}$ from the positive one to the negative one can be seen. A steep $E_{r}$ gradient is obtained with the radial transition. The radial point for the steepest $E_{r}$ gradient is defined by the parameter $\rho_{\mathrm{T}}$. Figure 1 (b) shows a self-consistent stationary profile of the turbulent heat diffusivity in the presence of the ZF effect (solid line). The turbulent diffusivity is clearly reduced in the core region in the presence of ZF effects compared with that in their absence. In the calculation here, we consider the radial variation of the turbulent particle diffusivity. The density increases because of the reduction in particle diffusivity in the presence of ZFs compared with that in their absence when we take the same value for $S_{0}$. Therefore, the value of the radial point for the $E_{r}$ transition, $\rho_{\mathrm{T}}$ in the presence of $\mathrm{ZF}$ effects differs from that in their absence. The radial point of $\rho_{\mathrm{T}}$ moves inside if the effect of ZFs is included in this calculation. The value of the reduced transport coefficient is close to that reported from LHD experiments [22]. The damping rate of ZFs is small in the electron root branch. The turbulent transport coefficient decreases when a strong positive radial electric field has formed. Outside the transition point $\rho_{\mathrm{T}}$, where the ion root branch appears, the damping rate increases and strong ZFs are not excited. Thus, a reduction in turbulent transport is not expected for the region $\rho>\rho_{\mathrm{T}}$ in this example. Figure 1 (c) shows the radial profiles of the sum of the turbulent heat diffusivity and the neoclassical heat diffusivity of electrons with (solid line) and without (dashed line) ZFs. A reduction of the same level as that in the turbulent transport profile appears with the effect of ZFs, because neoclassical 


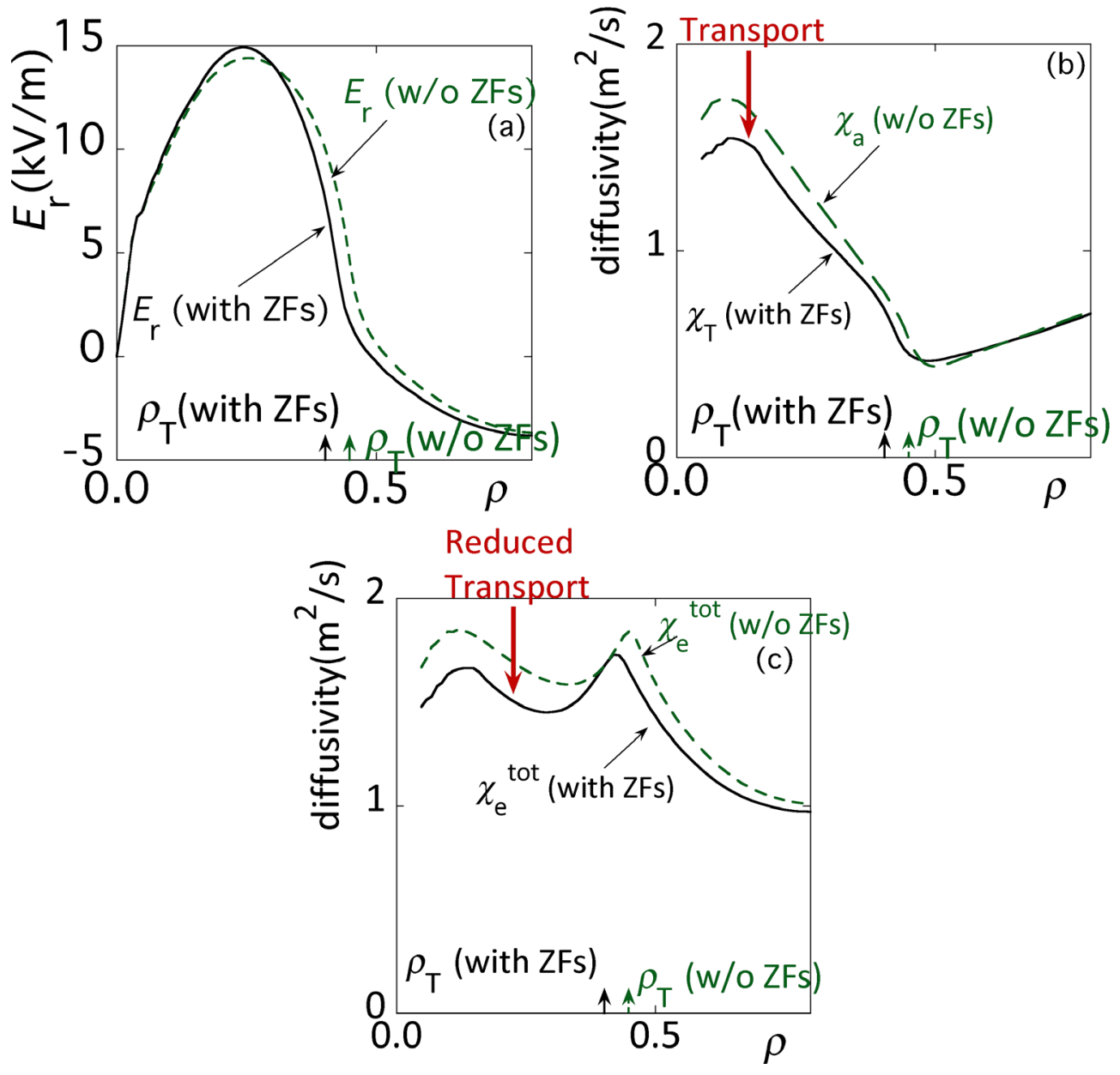

Fig. 1 (a) Profiles of the radial electric field without (dashed line) and with (solid line) zonal flows (ZFs). (b) Radial profiles of the heat diffusivities without ZFs, $\chi_{\mathrm{a}}$ (dashed line), and with ZFs, $\chi_{\mathrm{T}}$ (solid line). (c) Radial profiles of the total heat diffusivities without (dashed line) and with (solid line) ZFs.

transport is suppressed as the results of the strong positive $E_{r}$ in the core region of the e-ITB.

The excitation of ZFs depends strongly on the wave numbers of the microscopic fluctuations and the radial wave number of ZFs because the ZF excitation is determined by comparing $\chi_{\mathrm{a}}$ with the quantity $\chi_{\text {damp }}=$ $k_{\perp}^{2} q_{r}^{-2} k_{\theta}^{-2} v_{\text {damp }}$, where $q_{r}$ is the wave number of ZFs, and $k_{\theta}$ and $k_{\perp}$ are the poloidal and perpendicular wave numbers of the microscopic fluctuations, respectively. The value of $k_{\perp}^{2} q_{r}^{-2} k_{\theta}^{-2} \rho_{\mathrm{i}}^{-2} \sim 50\left(k_{\perp}^{2} q_{r}^{-2} \sim 6, k_{\theta} \rho_{\mathrm{i}} \sim 1 / 3\right)$ is chosen in this calculation. If the value of $k_{\theta} \rho_{\mathrm{i}}$ increases, the value of the criterion $\chi_{\text {damp }}$ for the excitation of ZFs decreases if the value of $k_{\perp} q_{r}$ is fixed. In this case, ZFs tend to be excited, and the turbulent transport will be reduced further.

\section{Results of the Analysis of IDB/SDC Plasmas}

The temporal response of the plasma to an additional particle source (particle fueling) is studied. Onedimensional transport analysis for an LHD-like plasma is performed using the modeled particle and heating sources described in Sec. 4. Figure 2 shows the dynamics of the plasma radial profiles for the density (a) and radial electric field (b) at the times 0,1 , and $12 \mathrm{~ms}$. The profiles at $0 \mathrm{~ms}$ (dotted line) represent the initial state used in the calculation. Dashed lines in Fig. 2 show the profiles at $1 \mathrm{~ms}$, when the pulse of the additional particle source $S_{\mathrm{p}}$ is switched off. Solid lines in Fig. 2 show the profiles at $12 \mathrm{~ms}$, illustrating self-generated dynamics. A temporal increase in the density at $1 \mathrm{~ms}$ after the initial conditions in the core region caused by the additional particle source $S_{\mathrm{p}}$ is shown in Fig. 2 (a). After $1 \mathrm{~ms}$, the density in the core region continues to increase slightly because an inward neoclassical transport occurs as a result of the positive gradient of the temperature profiles. At the end of the additional particle fueling ( $1 \mathrm{~ms}$ ), the gradient of $E_{r}$ is steeper owing to the effect of the additional particle source $S_{\mathrm{p}}$. We emphasize that a much steeper gradient in $E_{r}$ at the transition point $\rho_{\mathrm{T}}$ is generated at $12 \mathrm{~ms}$ after particle fueling. The parameter $\rho_{\mathrm{T}}$ represents the radial location of the steepest gradient of $E_{r}$ at $12 \mathrm{~ms}$. The $E_{r}$ gradient is strong enough to suppress 

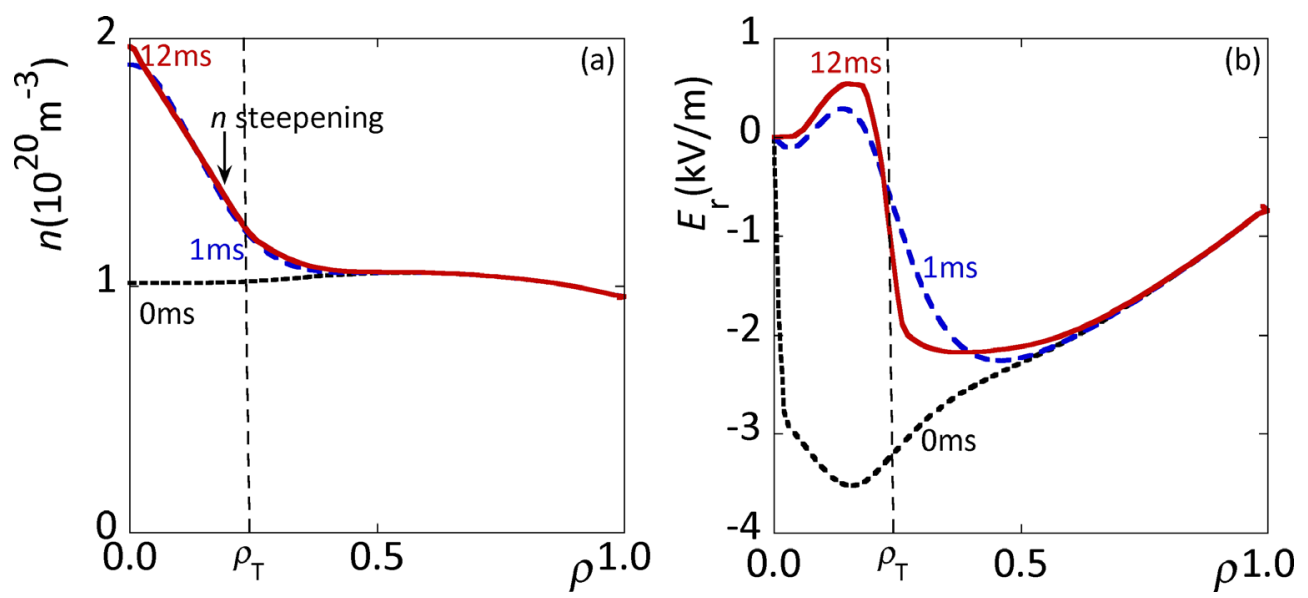

Fig. 2 Dynamics of the radial profiles of the density (a) and the radial electric field (b). Dotted, dashed, and solid lines show the states at the times 0,1 , and $12 \mathrm{~ms}$, respectively.

turbulent transport $E_{r}^{\prime} \simeq 10^{5} \mathrm{~V} / \mathrm{m}^{2}$ at $\rho=\rho_{\mathrm{T}}$. Therefore, an improvement is induced near the transition point. The dynamics of the profiles for the electron and ion temperature are also examined. The temperatures in the core region decrease from the initial condition because the density in this region increases owing to the additional particle source and the pressure is constant at this time scale $(\sim 12 \mathrm{~ms})$, which is shorter than the confinement time $(\sim 1 \mathrm{~s})$. The temperature gradient changes from negative values to less negative or positive values in the effective region of the additional particle fueling $S_{\mathrm{p}}$.

The mechanism why the gradient of $E_{r}$ steepens after the additional particle fueling is switched off is as follows. The value of $E_{r}$ given by the solution of the ambipolar condition is approximately written as

$$
E_{r}=T_{\mathrm{i}}\left(n^{\prime} / n+C_{\mathrm{i}} T_{\mathrm{i}}^{\prime} / T_{\mathrm{i}}\right) / e,
$$

where $C_{\mathrm{i}} \simeq 3$ in the parameter region of $n \geq 1 \times 10^{20} \mathrm{~m}^{-3}$ and $T_{\mathrm{e}} \simeq T_{\mathrm{i}}$. Note that the contributions to $E_{r}$ from the gradient of $T_{\mathrm{i}}$ are stronger than those from the gradient of $n$ by the factor $C_{\mathrm{i}}$. Because of the additional particle source $S_{\mathrm{p}}$, the value of $E_{r}^{\prime}$ changes. At $1 \mathrm{~ms}$, a steep $E_{r}$ gradient is obtained because the value of $T_{\mathrm{i}}^{\prime}$ increases in the effective region of the additional particle fueling $S_{\mathrm{p}}$. Next, we examine the self-generated dynamics of $E_{r}^{\prime}$. Even after particle fueling stops, the $E_{r}$ gradient continues to steepen. From $1 \mathrm{~ms}$ to $12 \mathrm{~ms}$ in the region $\rho<\rho_{\mathrm{T}}$, the density gradient decreases slightly and the ion temperature gradient increases. Because the contribution of the the ion temperature gradient to the ambipolar equation dominates the contribution of the density gradient, the value of $E_{r}$ becomes larger at $12 \mathrm{~ms}$ than at $1 \mathrm{~ms}$ in the region $\rho<\rho_{\mathrm{T}}$. Between $1 \mathrm{~ms}$ and $12 \mathrm{~ms}$ in the region $\rho>\rho_{\mathrm{T}}$, the density gradient increases slightly and the ion temperature gradient decreases. The value of $E_{r}$ is smaller at $12 \mathrm{~ms}$ than at $1 \mathrm{~ms}$ in the region $\rho>\rho_{\mathrm{T}}$. That is, even after the additional particle fueling ends, the value of $E_{r}$ in the region $\rho<\rho_{\mathrm{T}}$ evolves to in-

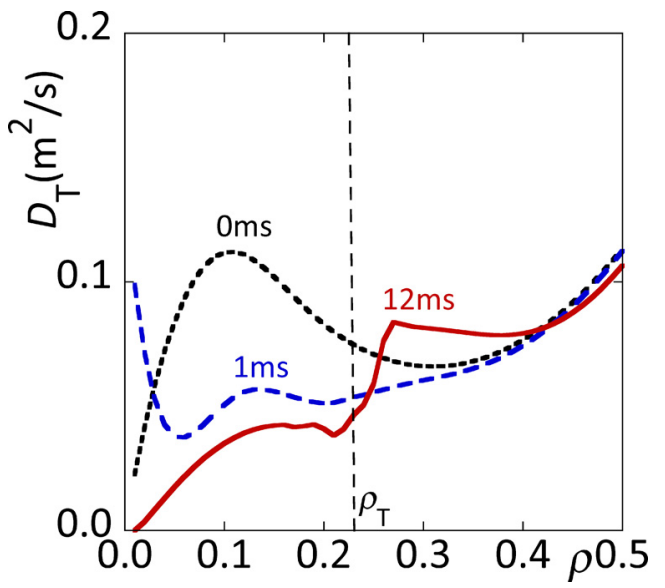

Fig. 3 Radial profiles of turbulent particle diffusivity $D_{\mathrm{T}}$ in the region $0<\rho<0.5$ at $0 \mathrm{~ms}$ (dotted line), $1 \mathrm{~ms}$ (dashed line), and $12 \mathrm{~ms}$ (solid line).

crease, whereas that outside $\left(\rho>\rho_{\mathrm{T}}\right)$ decreases because of the factor $C_{\mathrm{i}}(\simeq 3)$ in Eq. (1), which determines the ambipolar radial electric field. Thus, the strong shear of the radial electric field is self-organized at the transition point $\rho=\rho_{\mathrm{T}}$, after the additional particle source is switched off. When strong shear appears in $E_{r}$, the turbulent particle diffusivity is reduced, and the temperature gradient steepens. As a result, the radial electric field gradient is found to be much steeper due to the self-generated change.

Figure 3 shows a profile of the turbulent transport diffusivity at $12 \mathrm{~ms}$ (solid line) together with those at 0 and $1 \mathrm{~ms}$. The turbulent particle diffusivity is clearly reduced at the transition point $\rho_{\mathrm{T}}$ compared with that in the region $\rho>\rho_{\mathrm{T}}$ because of the strong $E_{r}$ gradient at $12 \mathrm{~ms}$. We confirm that the value of the turbulent particle diffusivity is much larger than that of the neoclassical particle diffusivity for $\rho<0.4$ in the parameter region examined here. At $12 \mathrm{~ms}$, the value of the turbulent particle diffusivity decreases for $\rho<\rho_{\mathrm{T}}$ and increases for $\rho>\rho_{\mathrm{T}}$ compared with 


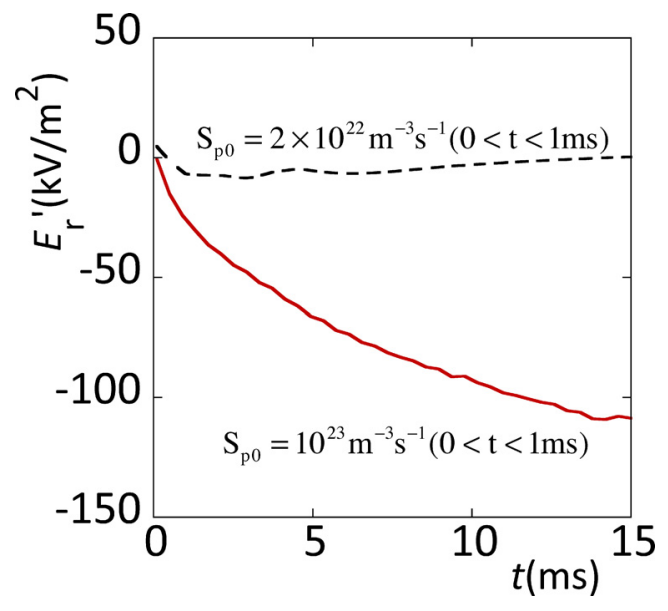

Fig. 4 Temporal evolution of the smallest value of $E_{r}^{\prime}$ in the region $0.15<\rho<0.25$ for $S_{\mathrm{p} 0}=2 \times 10^{22} \mathrm{~m}^{-3} \mathrm{~s}^{-1}$ (dashed line) and $S_{\mathrm{p} 0}=1 \times 10^{23} \mathrm{~m}^{-3} \mathrm{~s}^{-1}$ (solid line).

its value at $1 \mathrm{~ms}$. We clearly find a self-generated barrier at $12 \mathrm{~ms}$ in the radial profile of the turbulent particle diffusivity. A sharp change in the density gradient at $12 \mathrm{~ms}$ is observed. Therefore, a barrier with respect to particle transport in the density profile was obtained in Fig. 2 at $12 \mathrm{~ms}$.

We emphasize that the barrier formation continues after the additional particle source is switched off. The reduction in turbulent transport is much clearer in the region $\rho<\rho_{\mathrm{T}}$ after the additional particle source is switched off $(12 \mathrm{~ms})$ than at $1 \mathrm{~ms}$. Whether we can obtain a strong shear in the radial electric field depends on the value of $S_{\mathrm{p}}$, i.e., the amount of the additional particle source. We can examine the impact of the additional particle source on the self-generated shear of the radial electric field in the plasma dynamics. The temporal evolution of $E_{r}^{\prime}$ for $0.15<\rho<0.25$ is shown in Fig. 4. We show the cases of the large pellet fueling, $S_{\mathrm{p} 0}=1 \times 10^{23} \mathrm{~m}^{-3} \mathrm{~s}^{-1}$ (solid line), and the small pellet fueling, $S_{\mathrm{p} 0}=2 \times 10^{22} \mathrm{~m}^{-3} \mathrm{~s}^{-1}$ (dashed line) in Fig. 4. When $S_{\mathrm{p} 0}=1 \times 10^{23} \mathrm{~m}^{-3} \mathrm{~s}^{-1}$, the strong $E_{r}$ gradient that reduces turbulent transport evolves as selforganized, and we show that the typical time scale to obtain a strong $E_{r}$ gradient is around $10 \mathrm{~ms}$ in the parameter regime examined here. If we set a much smaller value for $S_{\mathrm{p} 0}, S_{\mathrm{p} 0}=2 \times 10^{22} \mathrm{~m}^{-3} \mathrm{~s}^{-1}$, the $E_{r}$ gradient increases with the additional particle source at $t=1 \mathrm{~ms}$. However, the $E_{r}$ gradient then smoothly decreases and returns to the initial state. This suggests a threshold value of $S_{\mathrm{p} 0}$ that affects the behavior of the shear of the radial electric field. The nature of the bifurcation in the plasma dynamics is studied. The dependence of $E_{r}^{\prime}$ on the magnitude of the particle fueling $S_{\mathrm{p} 0}$ is shown in Fig. 5 at $1 \mathrm{~ms}$ (open triangles) and $10 \mathrm{~ms}$ (closed circles). A continuous change in $E_{r}^{\prime}$ is shown at $1 \mathrm{~ms}$ in Fig. 5. At $10 \mathrm{~ms}$, a sharp change in $E_{r}^{\prime}$ due to the additional particle source appears clearly around $S_{\mathrm{p} 0}=5.0 \times 10^{22} \mathrm{~m}^{-3} \mathrm{~s}^{-1}$, marking the threshold value for

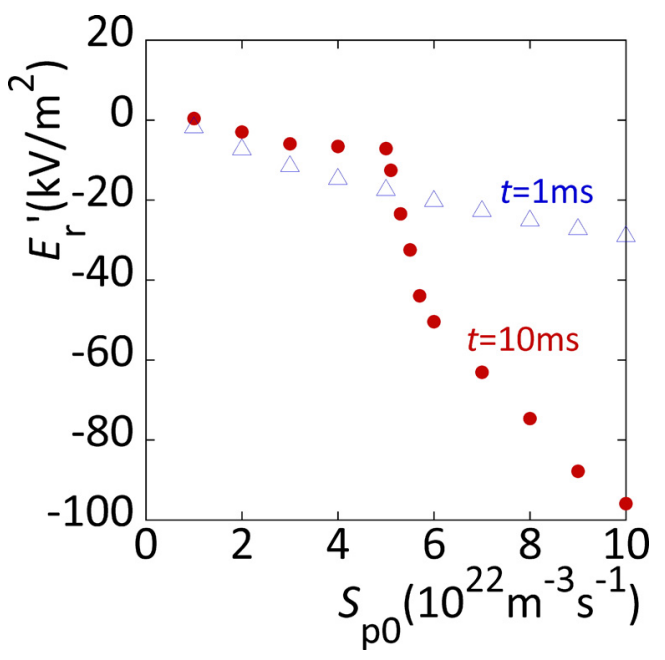

Fig. 5 Dependence of the radial electric field shear in the region $0.15<\rho<0.25$ on particle fueling $S_{\mathrm{p} 0}$ at $1 \mathrm{~ms}$ (open triangles) and $10 \mathrm{~ms}$ (closed circles).

$S_{\mathrm{p} 0}, S_{\mathrm{pth}}$. In the case $S_{\mathrm{p} 0}<S_{\mathrm{pth}}$, the conditions return to the initial state, which shows no improvement in transport after the additional particle source is switched off. If the value of $S_{\mathrm{p} 0}$ becomes larger and the relation $S_{\mathrm{p} 0}>S_{\mathrm{pth}}$ is satisfied, we can find the self-generated shear of the radial electric field in the plasma dynamics after the additional particle source is switched off. At $10 \mathrm{~ms}$, the shear of the radial electric field steepens, following the relation $E_{r}^{\prime}\left(S_{\mathrm{p} 0}\right)-E_{r}^{\prime}\left(S_{\mathrm{pth}}\right) \propto-\left(S_{\mathrm{p} 0}-S_{\mathrm{pth}}\right)^{0.43 \pm 0.10}$ for $S_{\mathrm{p} 0}>S_{\mathrm{pth}}$. This sharp change in $E_{r}^{\prime}$ depending on the additional particle source demonstrates the bifurcation of the plasma dynamics.

\section{Study of the Density Limit for the IDB}

To examine the density limit for thermal stability in the case of the IDB, we add a term for the radiation loss rate of the energy to the temporal equation for the electron temperature. Our study of the density limit considers only the case of the IDB, because the density limit will be a more critical issue in the case of the IDB than in the case of the e-ITB. The combined mechanism of the transport and the radiation loss of the energy is discussed. The form of the radiative loss rate was given in [23]. As an example, we take an oxygen as an impurity ions with $n_{\text {oxygen }}=0.01 n$, where $n_{\text {oxygen }}$ is the density of an oxygen. For simplicity, the density profile from [7] is used as the temporally fixed density profile for the IDB plasma in the calculation here. This density profile is approximated as $n(\rho)_{\mathrm{IDB}}=4.49 \times 10^{20}+3.77 \times 10^{21} \rho^{2}-2.64 \times 10^{22} \rho^{3}+3.90 \times$ $10^{22} \rho^{4}+4.05 \times 10^{21} \rho^{5}-4.28 \times 10^{22} \rho^{6}+2.08 \times 10^{22} \rho^{7} \mathrm{~m}^{-3}$. We examine the temporal evolution of the electron and ion temperatures and the profile of the radial electric field from the ambipolar condition during the typical confinement 

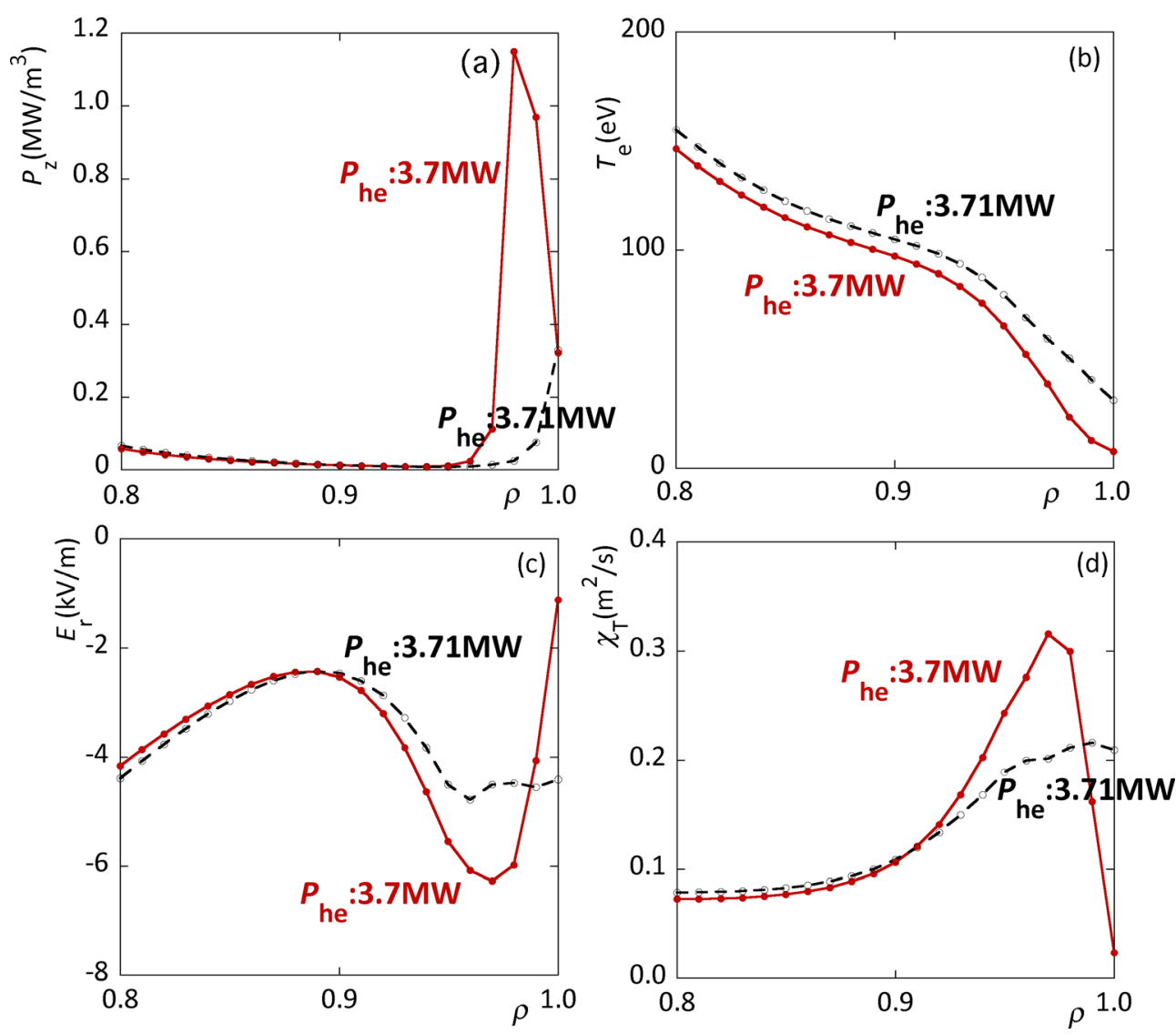

Fig. 6 Radial profiles in the region $0.8<\rho<1.0$ for an electron heating power of 3.7 MW (solid lines) and 3.71 MW (dashed lines). (a) Radial profiles of the radiative cooling rate. (b) Radial profiles of the electron temperature with the effect of the radiative cooling rate. (c) Radial profiles of the electric field. (d) Radial profiles of the turbulent heat diffusivity $\chi_{\mathrm{T}}$.

time ( 1s). Stationary profiles are obtained with electron heating power values of 3.7 MW (solid lines in Fig. 6) and 3.71 MW (dashed lines in Fig. 6) for $0.8<\rho<1.0$. Figure 6 (a) shows the radial profiles of the radiative cooling rate $P_{\mathrm{Z}}$. The profiles of the electron temperature are shown in Fig. 6(b). When the electron heating is $3.7 \mathrm{MW}$, the electron temperature decreases sharply near the edge. This is because the radiative loss rate rapidly increases at low temperatures $\left(T_{\mathrm{e}}<50 \mathrm{eV}\right)$. The effect of the ambipolar radial electric field is included in this calculation. The profiles of the ambipolar radial electric field are shown in Fig. 6(c). Even with this small difference in the electron heating power, the phenomena such as the transition near the edge in the $E_{r}$ profile appear only at an electron heating power of 3.7 MW. The value of $E_{r}$ changes from a negative value to close to zero near the edge. Therefore, the value of the turbulent heat diffusivity is significantly reduced because of the steep $E_{r}$ gradient near the edge in Fig. 6(d) when the electron heating power is $3.7 \mathrm{MW}$. If we choose a smaller value than $3.7 \mathrm{MW}$ for the electron heating power, we cannot obtain stationary profiles because of radiative loss near the edge. Next, we determine the minimum heating power needed to obtain a stationary solution in the presence of radiative loss and trans- port loss using a set of temporal transport equations for the electron and ion temperatures and the ambipolar radial electric field. We use temporally fixed density profiles, where $n(\rho)=\gamma n(\rho)_{\text {IDB }}$, and the parameter $\gamma$ takes the value $0.3 \leq \gamma \leq 1.0$ in this calculation. The value $P_{\min }$ of the electron heating power necessary to obtain a stationary state of plasmas is a function of the line-averaged density $\bar{n}_{\mathrm{c}}$. The condition $P_{\mathrm{e}} \geq P_{\min }$ can be shown as $\bar{n} \leq \bar{n}_{\mathrm{c}}$, where $P_{\mathrm{e}}$ is the electron heating power. The minimum value $P_{\min }$ is also a function of the line-averaged electron temperature $\bar{T}_{\mathrm{e}}$, and the condition $P_{\mathrm{e}} \geq P_{\min }$ can be shown as $\bar{T}_{\mathrm{e}} \geq \bar{T}_{\mathrm{ec}}$. The dependence of the critical density $\bar{n}_{\mathrm{c}}$ on the critical electron temperature $\bar{T}_{\text {ec }}$ is shown in Fig. 7 (a). Figure 7 (b) represents the dependence of the minimum electron heating power $P_{\min }$ on $\bar{T}_{\mathrm{ec}}$ and Fig. 7 (c) shows the dependence of the critical density $\bar{n}_{\mathrm{c}}$ on $P_{\min }$. The solid line in Fig. 7 (c) shows the critical density $\bar{n}_{\mathrm{c}}$ as a function of $P_{\text {min }}, \bar{n}_{\mathrm{c}} \propto P_{\text {min }}^{0.614 \pm 0.001}$. The Sudo scaling of the density limit from experimental results is shown in helical plasmas as $n_{\mathrm{c}}^{\mathrm{S}} \propto P^{0.5}$, where $P$ is the absorbed power [10]. The dependence of the critical density on the minimum electron heating power derived here in the case of the IDB is slightly stronger than the case of the Sudo scaling of the density limit. 

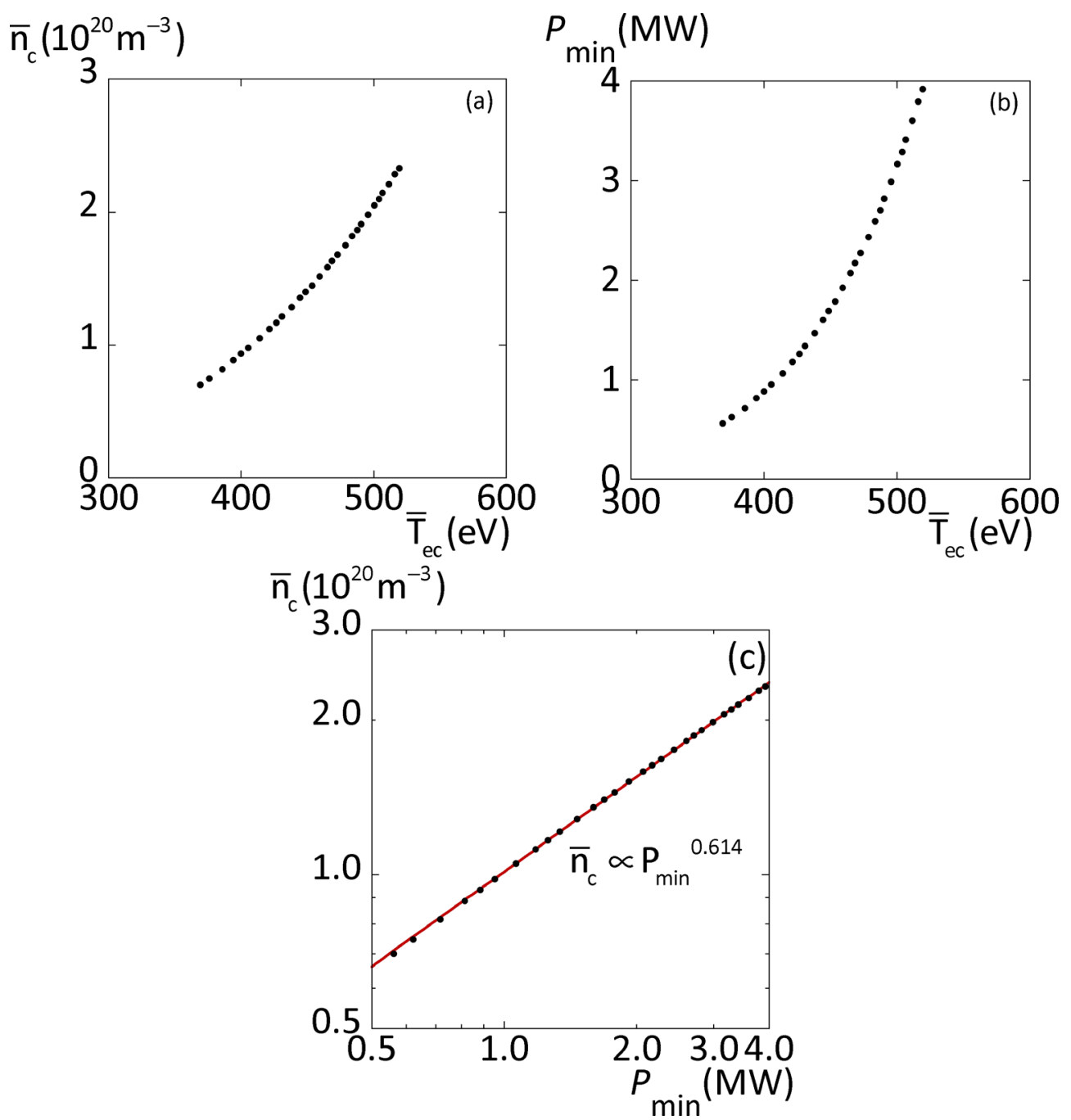

Fig. 7 (a) Dependence of the critical density (line-averaged) $\bar{n}_{\mathrm{c}}$ on the critical electron temperature (line-averaged) $\bar{T}_{\text {ec }}$. (b) Change in the minimum electron heating power $P_{\min }$ with the critical electron temperature $\bar{T}_{\mathrm{ec}}$. (c) Dependence of the line-averaged critical density $\bar{n}_{\mathrm{c}}$ on $P_{\min }$ in logarithmic scales on both the horizontal and vertical axes. Solid line approximately follows the relation $\bar{n}_{\mathrm{c}} \propto P_{\min }^{0.614}$.

\section{Summary}

Unified transport modeling was conducted with respect to the dynamics and radial structure of the density, temperature, and the electric field profiles in toroidal helical plasmas. The analysis is performed by use of the onedimensional transport equations. First, we studied the role of zonal flows in e-ITB formation in toroidal helical plasmas with radial variation in particle diffusivity. The small value of the damping rate of zonal flows suppresses turbulent transport. The results of transport code analysis show that the total heat diffusivity in the entire inner region is reduced. The effect of zonal flows on turbulent transport was investigated. A transport reduction was obtained in a wide region in conjunction with the e-ITB. This demonstrated that a change in the collisional damping of zonal flows can cause a transition in the turbulent transport. Next, we studied the temporal response of plasma to additional particle fueling in terms of IDB plasmas observed in the LHD. Af- ter particle ablation, the gradient of the radial electric field shows self-generated steepening due to plasma transport. The particle transport continues to decrease after particle fueling in the region $\rho<\rho_{\mathrm{T}}$ due to the shear in the electric field. Thus, the reduction in the turbulent particle diffusivity across the transition point $\rho_{\mathrm{T}}$ at $12 \mathrm{~ms}$ becomes clearer than that at the end of the particle fueling ( $1 \mathrm{~ms})$. The typical time scale to obtain the reduction in turbulent particle diffusivity was shown. This prediction explains theoretically the IDB plasmas in the LHD [7]. The sensitive dependence of the edge temperature profile on the electron heating was studied in the case of the IDB/SDC plasmas with radiative loss. The parameter dependence of the critical density on the electron temperature and the electron heating power was shown. To study the threshold value of $S_{\mathrm{p} 0}$ for obtaining a barrier with respect to particle transport, parameter survey of the calculation results is necessary. The investigation of other durations of particle fu- 
eling is planned. The parameter dependence of the critical density changes according to the impurity species and must be compared with the experimental results in detail. This will also be studied in the future.

The authors acknowledge Prof. A. Fukuyama for helpful suggestions related to the numerical methods. This work is partly supported by a Grants-in-Aid from the JSPS (No. 19360418) and the NIFS Collaborative Research Programs (NIFS08KLDD014 and NIFS08KNXN122).

[1] A. Fujisawa et al., Phys. Rev. Lett. 82, 2669 (1999).

[2] U. Strouth et al., Phys. Rev. Lett. 86, 5910 (2001).

[3] K. Ida et al., Phys. Rev. Lett. 91, 085003 (2003).

[4] T. Shimozuma et al., Plasma Phys. Control. Fusion 45, 1183 (2003).

[5] A. Fujisawa, Plasma Phys. Control. Fusion 45, R1 (2003).

[6] S. Toda and K. Itoh, Nucl. Fusion 47, 914 (2007).

[7] N. Ohyabu et al., Phys. Rev. Lett. 97, 055002 (2006).

[8] M. Greenwald et al., Nucl. Fusion 28, 2199 (1988).
[9] M.Z. Tokar et al., Plasma Phys. Control Fusion 37, A241 (1995).

[10] S. Sudo et al., Nucl. Fusion 30, 11 (1990).

[11] F. Wagner et al., Plasma Phys. Control. Fusion 36, A61 (1994).

[12] J. Miyazawa et al., Nucl. Fusion 48, 015003 (2008).

[13] K. Itoh, S.-I. Itoh and L. Giannone, J. Phys. Soc. Jpn 70, 3274 (2001).

[14] K.C. Shaing, Phys. Fluids 27, 1567 (1984).

[15] K.C. Shaing and J.D. Callen, Phys. Fluids 26, 3315 (1983).

[16] D.E. Hastings, Phys. Fluids 28, 334 (1985).

[17] K. Itoh et al., Plasma Phys. Control. Fusion 36, 123 (1994).

[18] K. Itoh et al., Plasma Phys. Control. Fusion 36, 279 (1994).

[19] P.H. Diamond et al., Plasma Phys. Control. Fusion 47, R35 (2005).

[20] K. Itoh et al., Phys. Plasmas 12, 062303 (2005).

[21] K. Itoh et al., Phys. Plasmas 14, 020702 (2007).

[22] O. Motojima et al., Nucl. Fusion 45, S255 (2005).

[23] D.E. Post and R.V. Jensen, Atomic Data Nucl. Data Tables 20, 397 (1977). 